Revista de Psicología de la PUCP. Vol. XII. No 2. 1994

\title{
ESTIMACION DE LA CIFRA VERDADERA DEL SUICIDIO EN ECUADOR ${ }^{1}$
}

\author{
Klaus-Dieter Gorenć ${ }^{2}$ Saúl Pacurucu ${ }^{3}$ Carlos Bruner ${ }^{4}$
}

Los registros estadísticos oficiales sobre el suicidio no son precisos, debido al subreporte de estos casos. Considerando que la cifra verdadera del suicidio está constituida por la cifra oficialy la oscura, se intentó estimar esta última utilizando una sencilla ecuación de imputación proporcional. Se analizaron los anuarios estadísticos bajo el supuesto que la categoría de muerte violenta con intención desconocida contendría a la cifra oscura. Mediante esta técnica indirecta fue posible establecer que el promedio porcentual de la cifra oscura del suicidio (1970-1974) se encuentra disminuido respecto a lo expuesto en la literatura.

Palabras claves: suicidio, adultos, Ecuador.

\section{Estimation of the true suicide in Ecuador}

The official statistical records on suicide are not reliable due to an under reporting of suicide cases. Considering that the true suicide count is the sum of the official record plus the obscure cipher, an attempt was made to estimate the latter using a simple proporcional imputation formula. The official statistical records were re-analysed under the following rational: the category of violent death caused by acccident or intention, but where it could not be established for certain, served as a reservoir that contained the obscure cipher. Using this indirect technique, it was possible to determine that the percentage mean of the obscure suicide cipher (1970-1974) is lower than the showed in the literature. Key words: suicide, adults, Ecuador.

1. El levantamiento secundario de datos fue auspiciado por el Centro de Rehabilitación para alcohólicos de Cuenca República de Ecuador. La Secretaría de Educación Médica de la Facultad de Medicina, Universidad Nacional Autónoma de México apoyó la transformación electrónica de los datos y el análisis estadístico.

2. Investigador nacional (México), Secretaría de Educación Médica. Circuito Interior, Edificio B 6to. piso, 04510 Ciudad Universitaria México, D.F.

3. Director del Posgrado de Psiquiatría, Universidad de Cuenca, Casilla 1023, Ciudad de Cuenca, Ecuador.

4. Investigador nacional (México), Departamento de Psicología Experimental, Facultad de Psicología, Universidad Autónoma de México. 

Confiar es bueno, pero controlar es mejor. Sobre el arte de controlar descansa el método científico, que no es infalible; sin embargo, utilizarlo con rigor, minimiza el sesgo implícito en la opinión subjetiva (Gorenć,1987; Gorenć y Beltrán 1988)(sentido común de Wellenreuther, 1982). Controlar indica mantener constante (o variar en forma sistemática) las variables exógenas con capacidad de desfigurar cierto fenómeno; por ejemplo, el suicidio, de tal manera que los efectos puedan ser extraídos del registro (anuario) estadístico (Gorenć, Herrera y Reynaga, 1986). Para cumplir con este requisito, se hace necesario estructurar una investigación dirigida a establecer qué variables muestran fuerza suficiente para ocasionar desplazamientos que no reflejen la actividad de la conducta suicida, sujeta al análisis. Este estudio no finaliza con exponer un catálogo de factores con sus diferentes pasos de penetración, sino indica mantenerlo corriendo en forma paralela al registro de los datos crudos en los anuarios, debido a que se presenta una variabilidad en el grado de influencia y relación entre y dentro de las variables a lo largo del proceso social. Esto, a nivel esquemático, apunta sobre tres aspectos integradores de lo que suele denominarse control experimental (Castro, 1984) (y no experimental). El primero se refiere a la manipulación de la variable independiente; por ejemplo, que únicamente sea el médico-legista el que tenga la facultad de establecer el veredicto de la causa de muerte violenta. El siguiente punto está orientado a minimizar la participación de las variables llamadas extrañas (Castro, 1984) o de confusión (Spector, 1982); lo cual, fue ejemplificado al describir el control de variables exógenas que debe practicarse desde el levantamiento primario de datos hasta su presentación al usuario. El tercer aspecto está dirigido al registro de los cambios en la variable dependiente (cifra del suicidio anual) que ocurren en función de las manipulaciones de las variables independientes; verbigracia, en México, recientemente se modificó el certificado de defunción, simplificándolo; este efecto proba- 
blemente se refleje sobre algunas causas de muerte (¿suicidio?), mientras que otras, menos susceptibles a los cambios, muestren únicamente desviaciones ligeras en relación a los registros anteriores. Si se confronta el deber ser con el ser de los registros de la mortalidad general (y la violenta, en particular), indicaría que todas las conclusiones (cuando se lograron establecer), basadas en este tipo de datos, estarían acosadas por las diversas fuentes de la invalidez interna y externa; es decir, respecto a la generalización de los resultados dentro y fuera de un estudio determinado (Castro, 1984; Gorenć, et al., 1986); esto sobre todo en lo que se refiere a la direccionalidad de la carga de las frecuencias y la orientación hipotética (Welz, 1979).

Por ello, los registros oficiales han recibido calificativos como satisfactores ilusorios (Stengel, 1961) o sinteticos (Wekstein, 1979); sin embargo, Wellhöfer (1981) seńala que efectivamente los registros están rodeados de limitaciones, que no han sido, hoy por hoy, demostradas empíricamente, que estaría apoyado por el panorama que se observa en la primera tabla donde se expusieron las opiniones de 29 expertos, pero ninguno de ellos demostró la influencia de estos factores en el subregistro de la cifra del suicidio, por ejemplo, el $17 \%$ expresa que se presenta oscilaciones debido a la confiabilidad del protocolo del levantamiento primario de datos (certificados de defunción), pero ninguno ha medido la precisión; de haberlo realizado, se hubieran percatado de que debe existir una relación lógica entre las definiciones y las construcciones (reactivos, preguntas, afirmaciones, etc.); es decir, el certificado de defunción debe ser válido y para ello se requiere también que sea congruente, preciso, objetivo y consistente; debido a que el certificado puede ser confiable, más no válido, pero para que muestre esta característica, tiene que ser confiable (Nadelsticher, 1983); conformando un binomio, que tiende a cambiar por la acción del tiempo, desactualizándose, siendo necesario afirmarlo periódicamente (Llanos y Gorenc', 1988).

La mayor carga se ubicó sobre las actitudes sociales frente a la forma alterna de morir, no restringiéndola, sino ocultándola; sin embargo, este factor carece de fundamento empírico. Luego entonces no sería posible hablar de culturas facilitadoras o inhibidoras, tan solo de culturas con menor y mayor cifra oscura; por ejemplo, en la amplia región ocupada por la civilización maya los suicidas estaban protegidos por la diosa Iztab 
(Gorenć, 1981), mientras que en la altiplanicie eran punibles, dificultando la comparabilidad de los índices entre ambas culturas. Esto señala que los factores descritos en esta tabla, limitan la acción de equiparar las cifras dentro y entre los países. Otra forma que influye sobre la comparación de las medidas de riesgo, está relacionada con la variación del denominador que corresponde a la población expuesta al riesgo del suicidio (Aburto, 1979; Guerrero, González y Medina, 1981): verbigracia, no fue posible comparar las tasas expuestas en dos publicaciones provenientes de la Organización Mundial de la Salud: en 1968 (WHO, 1986) el denominador estaba sujeto a la población > 15 años de edad. En 1975 (OMS), el cálculo se realizó en base a toda la población,probablemente por que las tasas del suicidio se contrastaron con las otras causas externas, que en caso de seguir con los lineamientos dictados en 1968 (WHO, 1968), las tasas del grupo control deberán estar relacionadas con el parámetro de edad mencionado.

Considerando el efecto de las variables exógenas apuntadas en la tabla anterior, éste se puede detectar sobre la magnitud del subregistro del suicidio; la literatura especializada refiere que la cifra oscura de esta conducta muestra una variación porcentual que oscila entre 25 a 50 , que tampoco se obtuvo a través de una investigación específica. $\mathrm{Al}$ respecto, Dublin (1963) informa que el intervalo propuesto fue una comunicación personal de un científico de la ciudad de Nueva York en 1963. De la costa oeste de los Estados Unidos de Norteamérica en 1986 (Shneidman, 1988) se comunicó que la cifra real del suicidio sería de 50,000 casos y no de 22,000 registrados en los anuarios estadísticos; esto se encuentra relacionado con la experiencia de un profesor de tanatología de la cuidad de Los Angeles, agregando que aún no existen estudios definitivos en este campo. En México, durante la primera mitad de la década de los ochenta se realizó el ejercicio de calcular el porcentaje de la cifra oscura del suicidio armando una técnica indirecta con una simple ecuación de atribución proporcional (Gorenc', 1988; Gorenć y Bruner, 1986; Gorenć y Bruner, 1988; Gorenć y Nadelsticher, 1985), mediante la cual, se obtuvieron resultados similares a los anotados por los dos expertos angloamericanos (Dublin, 1963; Shneidman, 1985), pero con una ventaja, ya que al estratificar las variables independientes se pudo establecer que la mayor carga porcentual de la cifra oscura comprendía a las mujeres de 60 a 64 años de edad que utilizaron para suicidarse otros procedimientos y por los no especificados en la 
苦 Tabla 1: Indicadores posiblemente involucrados en elevar la cifra oscura del suicidio según 29 autores (100\%)

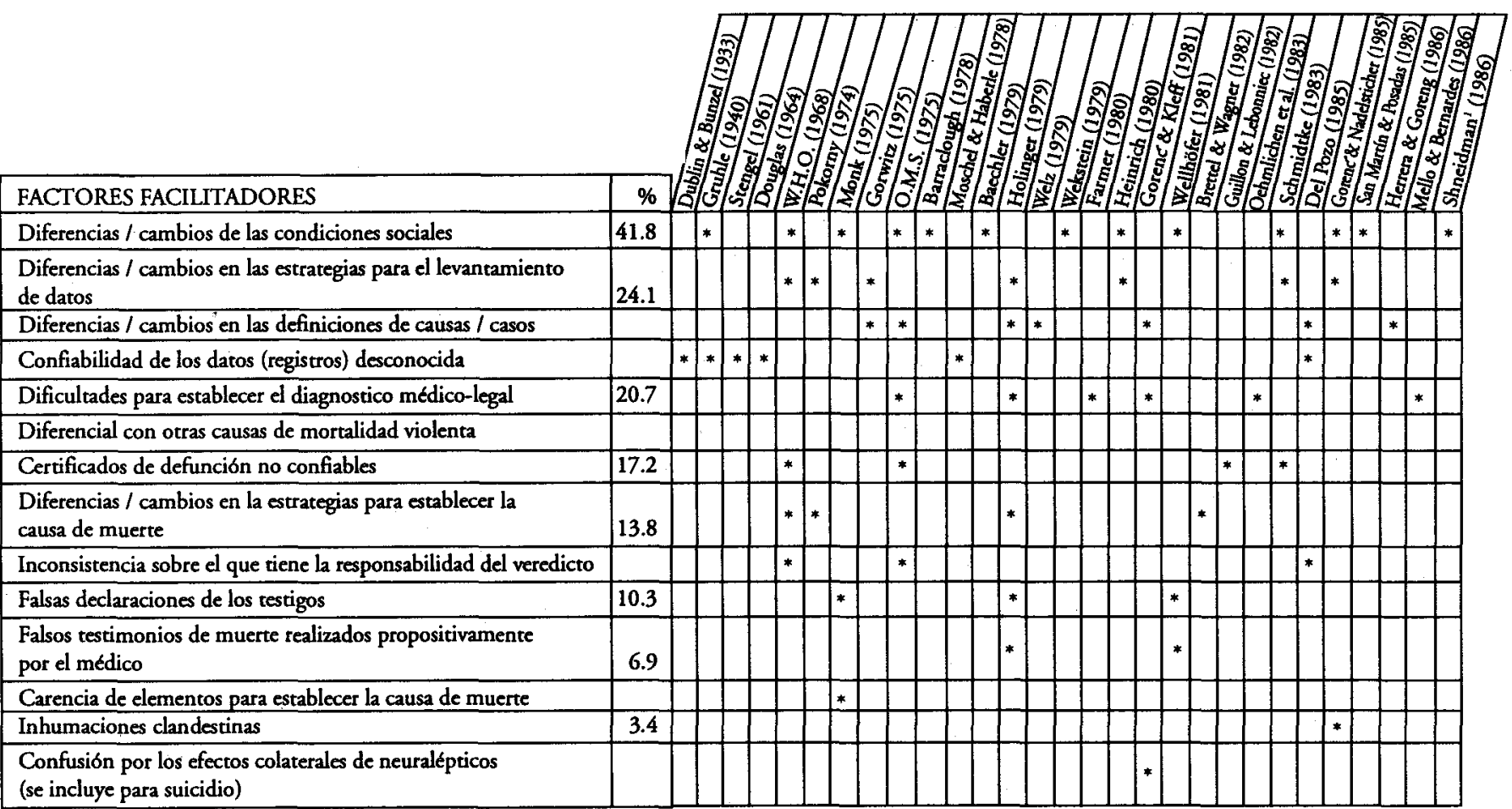

'Comunicación personal 
clasificación de la OMS respectiva, mientras que en los varones y casi independientemente de los grupos de edad, se obtuvo una tendencia de la cifra oscura a situarse en los métodos denominados pasivos (envenenamientos) (Gorenć, 1988). Estos resultados orientaron a externar que el suicidio se encuentra más tabuizado en las mujeres y que utilizan cada vez con mayor frecuencia los métodos que tradicionalmente caracterizaban al suicidio masculino; es decir, los métodos denominados duros (E953 a E957 en la segunda tabla), así como confirmar que la cifra verdadera del suicidio es proporcional al número de casos reportados en los registros estadísticos, que de acuerdo a Jakob (1979), una población con un reducido número de casos de suicidio, también presentará una cifra oscura reducida; lo opuesto también es válido.

Respecto a la imagen sombría de los registros del suicidio delineada en esta sección, Wellhöfer (1981) refiere que no le priva todo su valor; a pesar que reflejen en forma reducida y deformada esta conducta, muestran que existe ciertas estructuras y sobre todo estimula las investigaciones; verbigracia, Durkheim (1974) propuso la teoría de la desintegración social $\mathrm{y}$ anomia utilizando este tipo de datos, que intentan dar luz al contenido de las estructuras; al igual que en el presente estudio, donde se postuló, en base a lo externado por Dublin y Bunzel (1933), Jakob (1979) y Shneidman (1988), que la cifra oficial del suicidio registrada en los anuarios estadísticos; esto, controlando la influencia del sexo y los diferentes métodos.

\section{Material y métodos}

\section{Diseños}

Con el propósito de establecer, por un lado, la variabilidad del suicidio por espacio de un quinquenio (1970-1974) y por el otro, medir el impacto que pudiera tener la cifra oscura sobre la registrada, fue necesario articular dos diseños de tipo no experimental; es decir, de un grupo: el primero se denomina longitudinal (Spector, 1982) y consta únicamente de cinco observaciones, ya que fue la única serie de tiempo que no se encontraba interrumpida por anuarios estadísticos faltantes; este diseńo fue sometido a la acción de un pre y post test (Gorenć et al, 1986); esto es, la primera 
observación de cada ańo estudiado es la cifra registrada del suicidio; el tratamiento estaría conformado por la ecuación estructurada para extraer la cifra oscura de un reservorio o depósito destinado para los casos inclaros (AE-149) y agregarla a la cifra registrada, obteniendo así la segunda observación, que conforma la cifra verdadera. cifra registrada antes y después de la aplicación de la cifra oscura.

\section{Definición de caso}

Lindelius (1979) reportó que en Suecia (al igual que en Ecuador) hasta 1968 los casos dudosos habían sido clasificados como accidentes y a partir de 1969, estos casos se colocaron en un grupo especial: lesiones en las que se ignora si fueron accidentales o intencionalmente inflingidas (clave OMS: E980-E989; tabla 2), que funge a partir de ese ańo como depósito o reservorio de casos donde se desconoce si la muerte fue provocada por accidente, suicidio, homicidio o intervención legal (se abreviará como AE-149). La siguiente pregunta estaba orientada a establecer si existía una correspondencia entre los métodos de muerte de las cuatro modalidades de mortalidad violenta enumeradas previamente y E-149. Se encontró una completa sobreposición de los diez métodos entre AE-149 y el suicidio. Este resultado provocó que, para armar un engranaje operativo, era necesario considerar como casos sólo aquellos accidentes, homicidios e intervenciones legales que habían fallecido por uno de los diez métodos dispuestos en AE-149 y suicidio. Para extraer los casos de homicidio, accidente e intervención legal respecto a los diez métodos apuntados en la segunda tabla se utilizó la técnica de confiabilidad de retest (Carmines y Zeller, 1982) con dos levantadores de datos independientes y para controlar la correcta captura de los datos en la computadora, se estructuraron simples operaciones aritméticas de sumar las partes menos el total de cada evento (Gruhle, 1940).

Estimación de la cifra "verdadera" del suicidio por simple imputación proporcional.

El método indirecto de atribución proporcional se estructuró de la siguiente forma: después de extraer los casos de accidente, homicidio e intervención legal de acuerdo a los diez métodos de muerte que caracterizan 
Tabla 2: Semejanzas entre las subdivisiones de causas (métodos) de suicidio y los restantes causas de mortalidad violento

\begin{tabular}{|c|c|c|c|c|c|c|c|c|}
\hline $\begin{array}{l}\text { Lesiones en las que se ignore si } \\
\text { fueron accidental o intencio- } \\
\text { nalmente inflingidas }\end{array}$ & $\begin{array}{c}\text { Clave } \\
\text { OMS } \\
\left(y_{3}\right) \\
\end{array}$ & Suicidio & $\begin{array}{c}\text { Clave } \\
\text { OMS } \\
\left(y_{1}\right)\end{array}$ & $\begin{array}{l}\text { Homicidio y lesiones provoca- } \\
\text { das por orras personas }\end{array}$ & $\begin{array}{c}\text { Clave } \\
\text { OMES } \\
\left(y_{2}\right)\end{array}$ & Accidente & $\begin{array}{c}\text { Clave } \\
\text { OMS } \\
\left(y_{3}\right) \\
\end{array}$ & $\begin{array}{l}\text { Intervención } \\
\text { legal }\end{array}$ \\
\hline $\begin{array}{l}\text { Envenenamiento por sustancias } \\
\text { sólidas o lfquidos en el que se } \\
\text { ignore si fue accidental o inten- } \\
\text { cionalmente inflingidas }\end{array}$ & E980 & $\begin{array}{l}\text { Suicidio y envenenamiento } \\
\text { autoinflingido mediante sus- } \\
\text { tancias sólidas o líquidas }\end{array}$ & E.950 & $\begin{array}{l}\text { Envenenamiento intencional } \\
\text { provocado por orra persona }\end{array}$ & E962 & $\begin{array}{l}\text { Envenenamiento accidentales } \\
\text { por drogas y medicamentos } \\
\ldots \text { por otras sustancias sólidas } \\
\text { y liquidas }\end{array}$ & $\begin{array}{l}E 850 \\
E 859 \\
E 860 \\
E 869\end{array}$ & \\
\hline $\begin{array}{l}\text { Etventnamiento por gases de } \\
\text { uso doméstico }\end{array}$ & E981 & $\begin{array}{l}\text {... mediante gas para uso do- } \\
\text { mético }\end{array}$ & E951 & \multirow{2}{*}{ 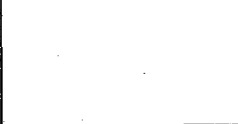 } & & \multirow[t]{2}{*}{... por gaves y vapores } & \multirow[t]{2}{*}{$\begin{array}{l}\text { E870 } \\
\text { E877 }\end{array}$} & . \\
\hline $\begin{array}{l}\text { Envenenamiento por otros ga- } \\
\text { ses... }\end{array}$ & E982 & ... mediante otros gases & E952 & & & & & $\begin{array}{l}\text { Lesión por intervención } \\
\text { legal con gases }\end{array}$ \\
\hline $\begin{array}{l}\text { Lesión por ahorcamiento y } \\
\text { estrangulación... }\end{array}$ & E983 & $\begin{array}{l}\text {... y lesión autoinflingida por } \\
\text { ahorcamiento, estrangulación y } \\
\text { sofocación }\end{array}$ & E953 & $\begin{array}{l}\text {... por ahorcamiento y entran- } \\
\text { gulación }\end{array}$ & E963 & $\begin{array}{l}\text { Aspinción e ingestión de aji- } \\
\text { mentos que provoquen obs- } \\
\text { trucción } \\
\text { Aspiración e ingestión de cual- } \\
\text { quier otro objeto que provoque } \\
\text { obstrucción o sofocación } \\
\text { Sofocación mecánica acciden- } \\
\text { tal }\end{array}$ & $\begin{array}{l}\text { E911 } \\
\text { E912 } \\
\text { E913 }\end{array}$ & . \\
\hline $\begin{array}{l}\text { Lesión por sumersión (ahoga- } \\
\text { miento) }\end{array}$ & E984 & $\begin{array}{l}\text {... por sumersión (ahoga- } \\
\text { miento) }\end{array}$ & E954 & $\begin{array}{l}\text {... por sumersión (ahoga- } \\
\text { miento) }\end{array}$ & E964 & $\begin{array}{l}\text { Ahogamiento y sumersión ac- } \\
\text { cidentales }\end{array}$ & E910 & \\
\hline $\begin{array}{l}\text { Lesión por arma de fuego y ex- } \\
\text { plosivos... }\end{array}$ & E985 & $\begin{array}{l}\text { \#. por arma de fuego y explo- } \\
\text { sivos }\end{array}$ & E955 & $\begin{array}{l}\text { Ataque con arma de fucgo y ex- } \\
\text { plosivos }\end{array}$ & E965 & $\begin{array}{l}\text {... causado por arma de fuego } \\
\text {... causado por material explo- } \\
\text { sivo }\end{array}$ & $\begin{array}{l}\text { E922 } \\
\text { E923 }\end{array}$ & $\begin{array}{l}\text {... con arma de fuego } \\
\ldots \text { con explosivos }\end{array}$ \\
\hline $\begin{array}{l}\text { Lesión por instrumentos cortan- } \\
\text { tes y punzantes... }\end{array}$ & E986 & $\begin{array}{l}\text {... por instrumentos cortantes } \\
\text { y punzantes }\end{array}$ & E956 & $\begin{array}{l}\text { Ataque con instrumentos cor- } \\
\text { tantes y punzantes }\end{array}$ & E966 & $\begin{array}{l}\text {... causado por instrumentos } \\
\text { cortantes y punzantes }\end{array}$ & E920 & $\begin{array}{l}\text {... con instrumentos cor- } \\
\text { tantes y punzantes }\end{array}$ \\
\hline $\begin{array}{l}\text { Lesión debida a precipitación } \\
\text { desde lugar elevado... }\end{array}$ & E987 & $\begin{array}{l}\text {... por precipitación desde lu- } \\
\text { gar clevado }\end{array}$ & E957 & $\begin{array}{l}\text {... por precipitación desde lu- } \\
\text { gar devado }\end{array}$ & E967 & Caldas accidentales & $\begin{array}{l}E 880 \\
\text { E887 }\end{array}$ & \\
\hline $\begin{array}{l}\text { Lesiones causadas por otros me- } \\
\text { dios especificados y por los no } \\
\text { especificados }\end{array}$ & E988 & $\begin{array}{l}\text {... por otros procedimientos y } \\
\text { los no especificados }\end{array}$ & E958 & $\begin{array}{l}\text { Atrque por otros procedimien- } \\
\text { tos especificados y los no espe- } \\
\text { cificzdos }\end{array}$ & E968 & $\begin{array}{l}\text { Orros accidentes y los no espe- } \\
\text { cificados }\end{array}$ & E929 & $\begin{array}{l}\text {... con el empleo de otros } \\
\text { medios especificos } \\
\ldots \text { con medias no expe- } \\
\text { cificados }\end{array}$ \\
\hline Effectos tardios... & E989 & $\begin{array}{l}\text { Efectos tardíos de lesiones } \\
\text { auroinflingidas }\end{array}$ & E959 & $\begin{array}{l}\text { Efectos tardios de lesiones } \\
\text { inflingidas intencionalmente } \\
\text { por orrz persona }\end{array}$ & E969 & $\begin{array}{l}\text { Efoctos tardios de un cnvene- } \\
\text { namiento accidental } \\
\text { Efectos tardios de una caida ac- } \\
\text { cidental } \\
\text { Efectos tandlos de orros acei- } \\
\text { dentes }\end{array}$ & $\begin{array}{l}\text { E942 } \\
\text { E943 } \\
\text { E946 }\end{array}$ & $\begin{array}{l}\text { Efectos tardlos de lesión } \\
\text { inflingida por interven- } \\
\text { ción legal }\end{array}$ \\
\hline
\end{tabular}


al AE-149 y suicidio, se estableció la diferencia porcentual entre accidente, suicidio, homicidio e intervención legal. Esta diferencia fue aplicada a la categoría de AE-149, que funcionó como un reservorio de la cifra oscura. Finalmente, de este depósito fueron extraídos proporcionalmente los casos que podrían corresponder al suicidio. Esto es, se pasó de un modelo estático de una cifra oscura del suicidio de 25 a $50 \%$ a uno dinámico, ya que se supuso que la cifra oscura del suicidio $\left(\mathrm{COY}_{1}\right)$ se encuentra en función de las condiciones en que se hallan los registros estadísticos (R) y las del depósito de los casos inclaros; lo cual, se puede esquematizar como $\mathrm{COy}_{1}=\mathrm{f}(\mathrm{R}, \mathrm{D})$ (Lewin, citado en Wellenreuther, 1982), que permitió el análisis de la cifra oscura en relación a las variables exógenas sexo y métodos de muerte. Este procedimiento puede describirse mediante una sencilla ecuación:

$$
\mathrm{COy}_{1,2,3, \ldots . . \mathrm{n}}=\left[\frac{\mathrm{y}_{1}}{\mathrm{x}-\mathrm{y}_{5}}\right] \mathrm{y}_{5}
$$

donde:

$$
\begin{aligned}
& \mathrm{CO}_{1} \\
& 1,2,3, \ldots \mathrm{n}= \begin{array}{l}
\text { cifra oscura del suicidio para el primer, segundo, } \\
\text { tercer, ...n años. }
\end{array} \\
& \mathrm{y}_{1}= \begin{array}{l}
\text { cifra registrada del suicidio en los anuarios } \\
\text { estadísticos }
\end{array} \\
& \mathrm{x}= \begin{array}{l}
\text { cifra de la muerte violenta registrada en los } \\
\text { anuarios estadísticos. } \\
\text { cifra en la que se ignora si las lesiones fueron } \\
\text { accidental o intencionalmente infligidas. }
\end{array}
\end{aligned}
$$

Esta ecuación indica que la cifra oscura del suicidio es una variable que depende de una exógena que es el registro oficial y una variable interviniente que es la magnitud del reservorio. Finalmente, para obtener la cifra verdadera del suicidio $\left(\mathrm{CV}_{1}\right)$ se lleva a cabo la suma respectiva $\left(\mathrm{y}_{1}+\mathrm{CO}_{1}\right)$

\section{Tasas}

Para comparar las frecuencias de un evento, llámese delito, enfermedad o muerte dentro de una población, habitualmente se utilizan las tasas; por 
ejemplo, la tasa del suicidio se calcula dividiendo el número de sujetos que cometieron suicidio entre el total de la población y el producto se multiplica por una constante (10 y sus múltiplos) (Monk, 1975). Cuando la tasa es calculada para la población general, pero en relación a un cierto fenómeno, se le denomina tasa de mortalidad específica; en este caso, por suicidio (Guerrero, et al., 1981; Reynaga, 1985). También se obtiene una tasa específica al relacionarse el evento a una característica de la población: $\geq 15$ ańos de edad, por sexo y otras variables más. Con el propósito de ampliar la comparación, las tasas se calcularon tomando en consideración a la población $\geq 15$ años de edad, para confrontar estos resultados a nivel internacional (Welz, 1979; WHO, 1968); además de que en el quinquenio analizado no se observó que esta conducta se presentara por debajo de la edad mencionada.

\section{Análisis}

Los datos obtenidos para estos cinco ańos fueron tabulados de acuerdo al formato de $\mathbf{8 0}$ columnas para utilizar el paquete estadístico para las ciencias sociales (SPSS), versión 8.3 (Nie y Hull, 1983). Para verificar los datos capturados así como las transformaciones realizadas, se enlistaron los casos, considerando dos puntos decimales para el formato (PRINT FORMAT y LIST CASES); para calcular las medidas de tendencia central y de dispersión, se utilizó el subprograma CONDESCRIPTIVE. Si bien es posible aplicar una prueba de significancia para la diferencia entre la cifra verdadera y la oficial, fue considerado en un principio sin sentido, debido a que ineludiblemente la cifra verdadera sería mayor que la registrada; sin embargo, y de acuerdo a Jakob (1979), no se esperaba obtener una cifra oscura del suicidio muy elevada ya que Ecuador se caracteriza por tener una tasa baja; por ello, se empleó la prueba estadística t-Student para muestras dependientes, siempre y cuando el porcentaje de la varianza fuera $\leq 50$.

\section{Resultados}

Independientemente del tiempo y ejercer control sobre alguna variable exógena, la cifra oscura del suicidio extraída del reservorio fue significativamente menor que la cifra registrada sin embargo, su efecto fue 
suficiente para que la cifra verdadera fuera mayor que la registrada y este producto no fue influido por el azar. Estos primeros resultados están relacionados con la baja carga porcentual de la cifra oscura, que se mantuvo por debajo del límite inferior marcado por la literatura (25\%) (Dublin, 1963). Las ligeras oscilaciones que se observan en las tasas, orientan a percibir el evento bastante estable y con un riesgo que se puede considerar bajo, en contraste a la de 15.2 que arrojó el Japón en 1970, pero superior a la registrada en Irlanda (1.8) durante este mismo ańo (OMS, 1975). Considerando el riesgo en la población $\geq 15$ años de edad y el bienio 1972/ 1973 (Welz,1979), una tasa promedio de 6.5 sitúa al Ecuador por arriba de la de Italia (5.8), nuevamente Irlanda (3.5), Grecia (3.4) y cerca de la que presentó Inglaterra (7.7); en comparación con México durante ese mismo quinquenio, la tasa promedio fue de 1.1 por 100,000 habitantes y 1.3 relacionada con la población $\geq 15$ años de edad (Gorenć, 1988).

\section{La influencia del sexo en la cifra "oscura"}

El panorama anotado al no ejercer control, se reproduce tanto en el sexo masculino y femenino, siendo más evidente en el primero; en todos los niveles de presentación de las cifras, en las mujeres, se ubica el menor efecto de 1 cifra oscura, que se ve reflejado en la apertura de la razón de la cifra oscura a favor (o en contra) de los varones, en contraste con las razones de la cifra registrada y la verdadera. En las tasas, la acción de la cifra oscura se atenúa en forma considerable e inclusive en el grupo de las mujeres, estas medidas de riesgo, entre ambos momentos (antes y después de la obtención de la cifra oscura e independientemente del denominador, se mantuvieron semejantes; lo cual, podría orientar a interpretar que la conducta suicida en el varón tiende a ocultarse con mayor fuerza; lo contrario, se presentó en el estudio mexicano: en la población masculina el intervalo de la cifra oscura varió entre 27 y $57 \%$, mientras que para la población femenina osciló entre 32 a 68\% (Gorenć y Bruner, 1988).

\section{La acción del sexo y los métodos sobre la cifra "oscura"}

La cuarta tabla se estructuró en relación a doce resoluciones y siete métodos; esto se originó a que entre los tres primeros métodos existe una enorme disparidad respecto al AE-149 y suicidio por un extremo y, por 
Tabla 3: Comparación de las cifras crudas y transformadas del suicidio en relación al tiempo (sin controlar y controlando el sexo)

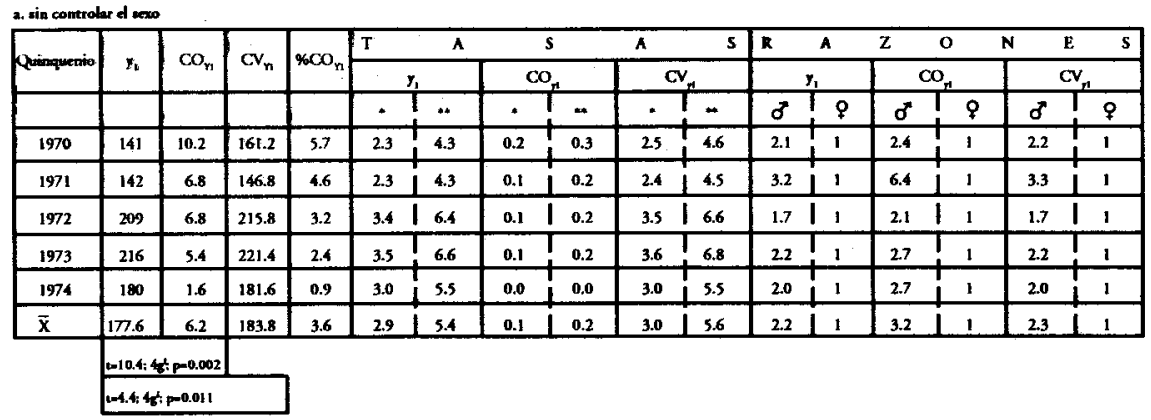

b. wero menculino

\begin{tabular}{|c|c|c|c|c|c|c|c|c|c|c|}
\hline 1970 & 96 & 7.1 & 103.1 & 6.9 & 3.2 & 6.0 & 0.2 & 0.4 & 3.4 & 6.4 \\
\hline 1971 & 108 & 5.8 & 113.8 & 5.1 & 3.6 & 6.7 & 0.2 & 0.4 & 3.7 & 7.1 \\
\hline 1972 & 131 & 4.5 & 135.5 & 3.3 & 4.3 & 8.2 & 0.1 & 0.3 & 4.5 & 8.4 \\
\hline 1973 & 149 & 3.9 & 152.9 & 2.5 & 4.9 & 9.3 & 0.1 & 0.2 & 5.0 & 9.5 \\
\hline 1974 & 120 & 1.1 & 121.1 & 0.9 & 3.9 & 7.5 & 0.0 & 0.1 & 40 & 7.5 \\
\hline$\overline{\mathbf{x}}$ & 120.8 & 4.5 & 125.3 & 3.8 & 3.9 & 7.5 & 0.1 & 0.3 & 4.1 & 7.8 \\
\hline & \multicolumn{2}{|c|}{$=11,9 ; 4 ;+1=0.000$} & & & & & & & & \\
\hline & \multicolumn{3}{|c|}{-0.5 . 4f:p-0.011 } & & & & & & & \\
\hline
\end{tabular}

\begin{tabular}{|c|c|c|c|c|c|c|c|c|c|c|}
\hline 1970 & 45 & 2.9 & 47.9 & 6.1 & 1.5 & 2.7 & 0.1 & 0.2 & 1.6 & 2.9 \\
\hline 1971 & 34 & 0.9 & 34.9 & 2.7 & 1.1 & 2.0 & 0.0 & 0.1 & 1.1 & 2.1 \\
\hline 1972 & 78 & 2.1 & 80.1 & 2.6 & 2.6 & 4.7 & 0.1 & 0.1 & 2.6 & 4.8 \\
\hline 1973 & 67 & 1.4 & 68.4 & 2.1 & 2.2 & 4.0 & 0.0 & 0.1 & 3.2 & 4.1 \\
\hline 1974 & 60 & 0.4 & 60.4 & 0.7 & 2.0 & 3.6 & 0.0 & 0.0 & 2.0 & 3.6 \\
\hline \multirow[t]{3}{*}{$\overline{\mathrm{x}}$} & 56.8 & 1.6 & 58.4 & 2.8 & 1.8 & 3.3 & 0.05 & 0.1 & 1.9 & 3.5 \\
\hline & \multicolumn{2}{|c|}{$\left(-7.1: 4 g^{6}: p=0.002\right.$} & & & & & & & & \\
\hline & \multicolumn{3}{|c|}{$1=3.5: 4 g^{2}+p=0.024$} & & & & & & & \\
\hline
\end{tabular}

- Tasas 100000 Habirantes

* Tasas 100 000 Habitantes $\geq 15$ afios de edad 
el otro, se encuentra un grupo constituido por las restantes formas de mortalidad violenta: accidente, homicidio e intervención legal (Cfr. tabla 2). En las denominadas Primera Resolución e independientemente del método utilizado para ocasionar la muerte, siempre se supuso que el rubro de AE149 contenía únicamente los casos inclaros de suicidio. La Segunda Resolución, el envenenamiento provocado intencionalmente por otra persona y donde no se encuentra especificado si el envenenamiento fue ocasionado por sustancias líquidas o sólidas, gas de uso doméstico o mediante otros gases fue incluido en la ecuación para extraer, de acuerdo a su respectiva carga, la cifra oscura del suicidio para cada uno de los tres primeros métodos. La Tercera Resolución se caracterizó por dejar afuera el homicidio del engranaje de la imputación proporcional. En esta tabla se omitieron las resoluciones y los métodos que mostraron una carga tan baja en los puntajes de la cifra oscura que se aproximaron fuertemente a la nulidad; éstas fueron:

a. Primera, Segunda y Tercera Resolución para el Segundo Método.

b. Primera, Segunda, Tercera, Cuarta y Quinta Resolución para el Tercer Método.

c. Quinto Método

d. Octavo Método

e. Décimo Método.

Mediante esta combinación de las variables exógenas, permite observar que la cifra oscura del suicidio en los varones descansa sobre un número más grande de métodos con mayor porcentajes de cifra oscura; el bajo porcentaje de esta cifra en las mujeres es sostenido por un método activo (Asberg, Träskman y Thoren, 1976); esto es, por el ahorcamiento, y en forma secundaria, por aquellas resoluciones y métodos que en la cuarta tabla se reflejan como cifras similares. Completando el cuadro nuevamente con la cifra oscura de los varones, ésta se basa principalmente en el primer método, que es considerado en la literatura (Asberg et al., 1976; Gorenć y Nadelsticher, 1985) como pasivo: envenenamiento por sustancia sólidas y líquidas; los siguientes pesos se encuentran ocupados por dos métodos activos o duros (Cfr. séptimo y noveno métodos en la tabla 2) y el último, 
Tabla 4: Diferencias del promedio (1970-1974) entre las cifras registradas del suicidio $\left(\mathrm{y}_{1}\right)$ cifras oscuras (CO) y cifras verdaderas (CV) respecto a cada uno de los métodos y controlando el sexo (prueba de significancia + - Student para muestras dependientes)

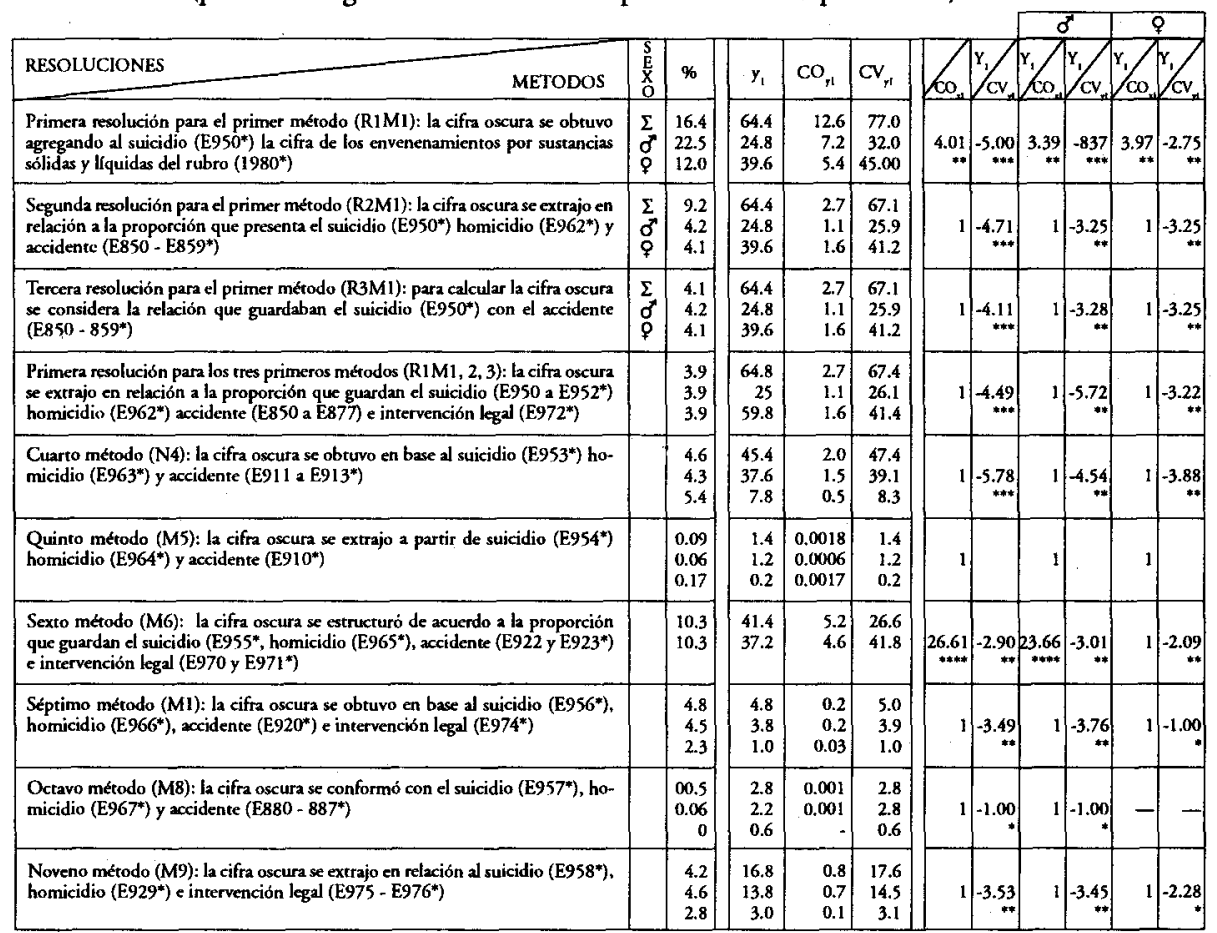

\begin{tabular}{|c|c|c|}
\hline $\begin{aligned} & * \mathrm{KS} \\
& * * \mathrm{p}<.05 \\
& * * * \mathrm{p}<.01 \\
& * 0 * * \mathrm{p}<.001\end{aligned}$ & siempre $g^{ \pm} \approx 4$ & $\begin{array}{l}\text { (*) y Cfr. tabla } 1 \\
\text { I varianza } 50 \% \text {, no siendo posible la interpretación } \\
\text { - no se presentó carga de frecuencias y por ello }(*) \text { idico que la } \mathrm{CV} \text { es semejante a la cifra registrada } \\
\text { Los signos negativos indican que las cifras oscuras y/o cifras verdaderas fueron mayores que las cifras regiseradas. }\end{array}$ \\
\hline
\end{tabular}


en el sexto método. Independientemente de las resoluciones y expuestos en la cuarta tabla se observa, en forma similar que en la tercera, un porcentaje de cifra oscura significativamente menor que la registrada, pero ese débil efecto fue lo suficientemente poderoso para elevar significativamente la cifra verdadera del suicidio.

\section{Discusión}

En el caso de Ecuador, el impacto de la cifra oscura del suicidio sobre la registrada se mantuvo por debajo del límite mínimo del $25 \%$ propuesto por Dublin (1963), confirmándose el planteamiento de Jakob (1979) respecto a que existe una relación entre el número de casos de suicidio y la carga de la cifra oscura correspondiente; por ejemplo, por cada caso de suicidio ecuatoriano, se presentaron 3.2 casos en México que se tradujo en un aumento porcentual en la cifra oscura de ese país, manteniendo constante la serie de tiempo; sin embargo, el riesgo muestra una imagen contraria (Gorenć, 1988), siendo invariablemente mayor en Ecuador (2.9/ 100000 habitantes y 5.4/100 000 habitantes $\geq 15$ años de edad sin cifra oscura y 3.0 y 5.6 con cifra oscura). En comparación con países con alto grado de desarrollo tecnológico, en 1970, por cada suicidio ocurrido en Ecuador, 6.6 se llevaron a cabo en Japón (OMS, 1975); considerando el bienio $1972 / 1973$ y un denominador caracterizado por 100,000 habitantes $\geq 15$ años de edad, la amplitud de la razón se reduce a 3.7 daneses (Welz, 1979) por cada ecuatoriano que comete suicidio; agregando la cifra oscura, no se observan cambios importantes en las razones: 1:6.0 en 1970 y 1:3.5 en el bienio anotado. En ambos momentos, la tasa del suicidio en Ecuador (con y sin cifra oscura) se puede considerar como baja y es semejante a la de los países europeos que se distinguen por un bajo desarrollo tecnológico: Irlanda, Grecia e Italia. Otra forma de notar el débil efecto de la cifra oscura en Ecuador, es estimar el parasuicidio en base a la frecuencia del suicidio. Stengel (1969) calculó que en promedio por cada suicidio consumado existen diez parasuicidios; esto es, en 1970,1410 personas presentaron una conducta parasuicida. Manteniendo constante el factor tiempo, la cifra oscura del suicidio comprendió a 10 casos más; es decir, alrededor de 100 casos más de parasuicidio; a los 1510 parasuicidas se requiere aumentar un 30\%, que según estimaciones de Welz (1979) respecto a la direccionalidad de la carga de frecuencia y la orientación hipotética y por el otro, la dis- 
crepancia entre los primeros métodos de muerte (M1, M2, y M3), originó la formación de dos bloques entre la mortalidad violenta, uno conformado por el suicidio y AE-149 y el otro, constituido por el accidente, homicidio e intervención legal; lo cual, condujo analizar los resultados de acuerdo a sus posibilidades de combinación, resultando doce supuestos para estimar la cifra oscura del suicidio; señalando que la estimación de la cifra oscura obtenida en base a las diferencias entre las cuatro formas de mortalidad violenta fue mejor que aquellas resoluciones marcadas por la unilateralidad; esto en relación a la oscilación de los porcentajes obtenidos por la décima segunda resolución: existe, al igual que en el estudio mexicano (Gorenć, 1988), un perfil similar entre las primeras formas de resolución mencionadas anteriormente y cuando los tres primeros métodos fueron analizados bajo su forma genérica; con lo que cabría señalar que estas formas de resolución para extraer la cifra oscura del suicidio, pueden considerarse como adecuadas, cuando se planea un estudio con derivación hipotética.

La fórmula de simple imputación proporcional propuesta, únicamente refleja el comportamiento que guardan entre sí las frecuencias de la mortalidad violenta. Llegado este momento, fue necesario operacionalizarla y formalmente funcionó; surgiendo acto continuo la siguiente pregunta ¿realmente se estimaba la cifra oscura del suicidio? Para responder, habría que proponer algunos ejercicios; por ejemplo, con aquellos resultados basados en un levantamiento secundario de datos, utilizando como fuente las actas de defunción de los casos que fueron incluidos en $\mathrm{y}_{5}$. Se trata de una propuesta bastante sencilla, ya que para llevar a cabo una reclasificación de los casos confusos, ésta debe partir de una definición de caso; verbigracia, a través de un formulario que contenga un número de variables, que podrían caracterizar, mediante una combinación algebraica cada una de las cuatro causas estaría estructurada de acuerdo al porcentaje de sobreposición entre los expertos $(\geq 50 \%)$. Es cuando se podría iniciar el trabajo de reclasificación de los casos. En esta labor deberían participar, por lo menos, dos clasificadores de casos cuyos resultados deberían ser entrecruzados para medir el índice de coincidencia; y, sólo se tomarían como casos aquellos que hubieran sido reclasificados correctamente por ambas personas. Estos resultados serían, entonces, contrastados con los obtenidos en esta investigación para medir la sobreposición de ambos estudios y así establecer la efectividad de la fórmula. Pero al contar ya con 
un instrumento válido y confiable, capaz de ubicar en forma correcta (con error conocido) los casos de defunción ocurridos en forma violenta, la ecuación resultaría ser trivial o posiblemente se emplearía para reclasificar el cúmulo de casos (supuestamente minoría) que no pudieran ser encasillados por el instrumento; pero como comprendería a una minoría de casos difíciles, y partiendo del principio que números pequeños no afectan a cifras grandes, la fórmula no tendría justificación y sería desechada. Por lo cual, mientras no se tenga una mejor alternativa, la ecuación podría usarse para aproximarse a una cifra más realista que la presentada en los anuarios estadísticos.

El panorama exhibido en la primera tabla, señala la falta de control de las posibles variables intervinientes, que va desde el llenado de las actas de defunción hasta la publicación del anuario estadístico. Respecto a esta cadena de procesamiento de datos, algunos especialistas (Gorenć y Nadelsticher, 1985; Lettieri y Nehemkis, 1974; Mello y Bernardes, 1986) entre otros) concuerdan que el problema debe situar en el protocolo del levantamiento primario de datos: el certificado de defunción, que está sujeto a las más variadas manipulaciones; por ello, se hace evidente la necesidad de estructurar actas estandarizadas capaces de excluir el sesgo de la información y que contenga todas las posibles alternativas para establecer las causas de defunción en base a un criterio que, estandarizado mediante un algoritmo computarizado, permita excluir los datos irrelevantes; en forma similar como se ha hecho en otro tipo de pruebas diagnósticas (Robins et al., 1981). Además debería estar estructurada en forma sencilla para que también los legos; por ejemplo, los agentes provinciales o municipales puedan vertir la información necesaria para establecer la causa de la mortalidad. El tiempo de llenado debería ser también mínimo, así como tener un costo bajo. Obviamente se requeriría del control sistemático; verbigracia, para cada defunción seleccionada al azar, un doble registro con levantadores de datos independientes con el fin de garantizar las medidas de seguridad a través del tiempo. Como la información estaría capturada en cinta y al día, no se necesitaría de las publicaciones periódicas y el usuario sólo tendría que sentarse frente a la terminal para buscar datos y enviarlos a la impresora e inclusive permitiría realizar, directamente y sin pérdida de riempo, (captura de los anuarios estadísticos a la cinta) el análisis estadístico con datos válidos y confiables. Con ello, desde el elevamiento 
primario de datos hasta la utilización de los mismos estaría altamente simplificado, debido a que la información podría llegar vía terminal, de cada servicio coordinador del Ministerio de Salud Pública, al archivo central; además, cada provincia tendría su propio banco de datos, que apoyaría la política de descentralización (Gorenć y Nadelsticher, 1985).

Agradecimientos

Los autores expresan su gratitud al Licenciado en Derecho Manuel Antonio Zurita Oropeza, Procurador General de Justicia del Gobierno del Estado de Tabasco por haber apoyado la realización de este estudio; extendemos nuestro agradecimiento al Maestro en Ciencias José Luis Barajas Quiles, Administrador de Base de Datos del Departamento de Apoyo Técnico de la Universidad Autónoma Metropolitana por habernos asesorado y facilitado el acceso al área de terminales y a la mencionada Casa Abierta al Tiempo por haber cedido amablemente tiempo de cómputo. Nuestro agradecimiento también está dirigido a la señora Margarita Estrada Gorenć quien realizó la revisión de estilo así como a Jadzia y Karel Gorenć por haber diseñado las tablas y participado en el test-retest de los datos respectivamente.

\section{Referencias}

Aburto, C. (1979). Elementos de bioestadística. México: Fondo Educativo Interameriano.

Asberg, M., Träskman, L. y Thoren, P. (1976). 5-HIAA in the cerebrospinal fluid. Arch. Gen.Psychiat, 33, 1193.

Baechler, J. (1979). Suicides. Nueva York: Harper Colophon Books, pp. 8-37.

Barraclough, B. (1979). The different incidence of suicide in Eire and in England and Wales. Brit. J. Psychiat, 132: 36.

Carmines, E. G. y Zeller, R.A. (1982). Reliability and validity assessment. Sage University Paper series on Quantitative Applications in the Social Science (07, Serial N017).

Castro, L. (1984). Diseño experimental sin estadística. México: Trillas.

Del Pozo, T. (1985). El suicidio en el Perú: algunos aspectos del registro.

Revista Neuro-Psiquiatría, 5, 149-150.

Douglas, J. (1967). The social meanings of suicide. Princentown: University Press. 
Dublin, L. I. (1963). Suicide: A sociological and statistical study. Nueva York: Ronald Press.

Dublin, L. I. y Bunzel, B. (1933). To be or not to be. Nueva York: Random House.

Durkheim, E. (1974). El suicidio. México, D.F.: Universidad Nacional Autónoma de México.

Farmer, R.D.T. (1980). The relation between suicide and parasuicide. En

R.D.T. Farmer y S. Hirsch (Eds.), The suicide syndrome (pp. 19-37).

London: Croom Helm.

Gorenc', K.-D. (1981). Der suizid in den bayerischen Bezirkskrankenhäusern.

Empirische Untersuchung. Tesis doctoral. Universidad de Munich.

Gorenc', K.-D. (1987). Prefacio. En Medicina Forense, Criminología y

Criminística: Un análisis retro y prospectivo (pp.15-18). Villahermosa:

Editorial Procuraduría General de Justicia del Gobierno del Estado de Tabasco.

Gorenć, K.-D. (1988). Tengo derecho a tener un último secreto... Acta médica.

Gorenć, K.-D. y Beltrán, J.E. (1988). El problema del alcoholismo en Tabasco. Villahermosa: Editorial Procuraduría General de Justicia del Gobierno del Estado de Tabasco.

Gorenć, K.D. y Bruner, C.A. (1986). La estimación de la cifra oscura del suicidio usando el método indirecto. En R. Díaz Guerrero (Ed.), La psicología social en México (pp. 547- 551). México: Ed. Asociación de Psicología Social.

Gorenć, K.-D. y Bruner, C.A. (1988). Technical note. The estimation of the "obscure" suicide rate by simple proportional imputation. Revista ILANUD.

Gorenc', K.-D., Herrera, M.E. y Reynaga, J. (1986). Manual de diseños de investigación. Series Manuales y Cursos del Instituto Nacional de Ciencias Penales (3).

Gorenć, K.-D. y Kleff, F., (1981). Selbstmord und Selbstmordversuch in psychiatrischen krankenhäusern. En: R. Welz y H. Pohlmeier (Eds.), Selbstmordhandlungen (pp. 187-210). Weinheim y Basilea: Beltz Verlag. Gorenć, K.-D. y Nadelsticher, A. (1985). Cifra oscura del suicidio en México (1969-1979). Cuadernos del Instituto Nacional de Ciencias Penales, 22, 95-96. 
Gruhle, H. W. (1940). Selbstmord. Stuttgart: Thieme Verlag.

Guerrero, R., González, C.L. y Medina, E. (1981). Epidemiologia. México,

D.F.: Fondo Educativo Ineramericano.

Guillon, C. y LeBonniec, Y. (1982). Suicide, mode d'emploi. París: Editions Alain Moreau.

Heinrich, K. (1980). Epidemiologische Faktoren der Suizidalität. Dtsch. med. Wschr, 105, 900.

Herrera, M.E. y Gorenć, K-D. (1986). El sexo y la edad como factores intervinientes del suicidio y las otras causas de mortalidad violenta. Rev. Méx. Jus., 3, 71.

Holinger, P.C. (1979). Violent Deaths Among The Young. Recent trends in suicide, homice, and accidents. Am. J. Psychiat, 136, 144.

Jakob, O.(1979). Aspekte der todesursachenstatistik 1877-1977 des genosischen statistischen Amtes. Tesis doctoral. Universidad de Zurich.

Lettieri,, D.J. y Nehemkis, A.M. (1974). A socio-clinical scale for certifying mode of death. En A.T.Beck, H.L.P. Resniky D.J. Letierri (Eds.), The Prediction of Suicide (pp. 119-140). Maryland: Charles Press Publications.

Lindelius, R. (1979) Trends in suicide in Sweden 1749-1975. Acta psychiat. scand., 60, 295.

Llanos, R. y Gorenć, K.-D.(1988). La prueba de Alcoholismo Munich (MALT) en Perú: Indicadores empíricos y sus errores en la medición. Revista de Neuro-psiquiatria.

Mello, M.H: y Bernardes, M. (1986). Mortes violents en Menores de 15 anos no Brasil. Bol. Of. Sanit. Panam.,6, 590.

Monk, M. (1975). Epidemiology. En S. Oerlin (Ed.), A handbook for the Study of Suicide (pp. 185-212). London: Oxford University Press.

Moschel, G. y Häberle, H. (1978). Selbstmord und seine sozialräumliche Bedingungen in Mannheim. En: H. Häfner, (Ed.), Psychiatrische Epidemiologie (pp. 59-80). Berlín: Springer-Verlag.

Nadelsticher, A. (1983). Técnicas para la construcción de cuestionarios de actitudes y opción múltiple. Series Manuales y Cursos del Instituto Nacional de Ciencias Penales (11).

Nie, N.H. y Hull, C.H. (1983). SPSSS-9 Statistikprogrammsystem für die Sozialwissenschaften. Stuttgart: Fischer Verlag.

Oehmlichen, M., Staak, M.M., Schmidt, V., Baedeke, Ch. y Sternus, K.- 
S. (1986). Leichenschau und Obduktion bei tod im Strassenverkehr. Deutsches Arzteblatt, 13, 873.

Organización Mundial de la Salud (1975). Comparabilidad de las Estadísticas sobre el Suicidio. Crónica O.M.S., 29, 201-207.

Pokorny, A.D. (1974). A scheme for classifying suicidal behaviors. En:

A.T. Beck, H.L.P. Resnik y D.J. Letierri (Eds.). The prediction of suicide (pp. 29-44). Maryland: Charles Press Pub.

Reynaga, J. (1985). Epidemiología II: Secuenica del quehacer epidemiológico. Cuaderno O.P.S., 5, 10.

Robins, L.M., Helzer, J.E., Croughan, J., y Ratcliff, K.S. (1981). Diagnostic interviwe Schedule. Arch. Gen Psychiat,38,381.

Schmidtke, A. (1981). Entwicklung der Häufigkeit suizidaler Handlungen im Kindes un Jungendalter in der B.R.D. Kinderarzt, 12, 697.

Schmidtke, A. (1983). Folgen Suizidziffern Zeitreihenmodellen?. En H. Pohlmeier, A. Schmidtke y R. Welz (Eds.). Suizidales Verhalten ( $\mathrm{p} 89$ 102). Regensburg: Roderer Verlag.

Schmidtke, A., Bangert, H., Blobel, R. y Remelius, B. (1980). Suizid un Selbstbeschädigung im Strafollzug. Artículo presentado en Bonn.

Shneidman, E. (1988). Comunicación personal.

Spector, P. E. (1982). Research Designs. Sage University Paper Series on

Quantitative Applications in the Social Sciences (07, Serial $N^{\circ}$ 023).

Stengel, E. (1961). Selbstmord und Selbstmord versuch. En H.W. Gruhle,

R. Jung, W. Mayer-Gross, W. y Müller (Eds), Psychiatrie der Gegennart

(pp. 51-74). Berlin, Heidelberg y Nueva York: Springer-Verlag.

Stengel, E. (1969). Selbstmord und Selbstmordversuch. Frankfurt/Main:

Fischer Verlag.

Wekstein, L. (1979). Handbook of suicidology. Nueva York: Bruner/Mazel Publications.

Wellhöfer, P. R. (1981). Selbstmord und Seibstnordversuch. Stuttgart: Fischer Verlag.

Welleneuther, M. (1982). Grundkurs: Empirische Forschungsmethoden. Königstein/Ts: Athenäum.

Welz, R. (1979). Selbstmordversuche in städtischen Lebensumweiten.

Weinheim: Beltz Verlag.

World Health Organization (1968). Prevention of suicide. WHO: Ginebra. 